This is a post-peer-review, pre-copyedit version of a paper published in New Trends in Mechanism and Machine Science. Mechanisms and Machine Science, vol 43 pp. 173-180 (2017) Springer, Cham. The final authenticated version is available online at: http://dx.doi.org/10.1007/978-3-319-44156-6_18.

Chapter 1

\title{
Kinematic Analysis of a Continuum Parallel Robot
}

\author{
Oscar Altuzarra $^{1}$, Mikel Diez ${ }^{1}$, Javier Corral ${ }^{1}$, Gennaro Teoli ${ }^{2}$ and Marco \\ Ceccarelli ${ }^{2}$ \\ ${ }^{1}$ University of the Basque Country UPV/EHU, Spain, e-mail: \\ oscar.altuzarra@ehu.eus \\ ${ }^{2}$ University of Cassino and South Latium, Italy.e-mail: ceccarelli@unicas.it
}

\begin{abstract}
Continuum Parallel Robots are mechanical devices with closed loops where kinematic pairs have been eliminated and motion is obtained by large deformations of certain elements. Most compliant mechanisms use notches in thick elements to produce the effect of kinematic pairs. A few are designed so that slender elements can deform and produce the desired motion. Some microelectromechanical systems have used this principle to create bistable planar mechanisms. The purpose of this work is to extend such principle in the field of macro mechanisms for manipulation. The aim is to design the counterparts to some classical parallel manipulators solving the corresponding kinematic problems. In doing this, the authors will have to work out the most efficient way to solve a position problem where geometry and forces are involved. Such compliant mechanisms could be combined in the future with tensegrity systems to enhance the available workspace. In this first report we will focus on the simplest planar parallel mechanism of two degrees of freedom.
\end{abstract}

Key words: Parallel Continuum Robot, Compliant Mechanism, Kinematic Analysis, Experimental Mechanics.

\subsection{Introduction}

Continuum Parallel Robots have been designed and studied with some success recently, either as compliant mechanisms [1,2] or soft robot systems [7]. However, apart from classical texts on the subject of nonlinear deformations such as [3], or a seminal work on compliant mechanisms such as [4], there is not much academic literature on the subject of the kinematics problems to be solved in such closed loop devices using analytical approaches. Nevertheless, some of the work done in MEMS devices can help in the process $[5,6]$.

The purpose of the work reported here is to establish an analysis frame where the systematic kinematic analysis of parallel devices that make use of flexible elements deforming in a nonlinear extent can be established. This is the first step in such journey, where starting from the classical analysis of the elastica of a bar clamped at both extremes that makes use of the elliptic integrals, we have solved a planar parallel device with two degrees of freedom analytically. The key issue is the consideration of the force equilibrium at the kinematic stage of the analysis. 


\subsection{Fundamentals of Flexible Bars Clamped at Both Ends}

A slender element under flexion in a plane acquires the form of a planar curve as in Fig. 1.1. Navier-Bernouilli hypothesis assumes that its cross sections remain planar and perpendicular to the bent curve. Bernoulli-Euler law establishes that the bending moment $M$ at a point is expressed proportional to the curvature $\kappa$ as:

$$
\kappa=\frac{d \theta}{d s}=\frac{M}{E I}
$$

where $E$ is the Young modulus and $I$ is the moment of the cross section about the neutral axis. Focusing on the case of a bar clamped at both extremes, we define for a

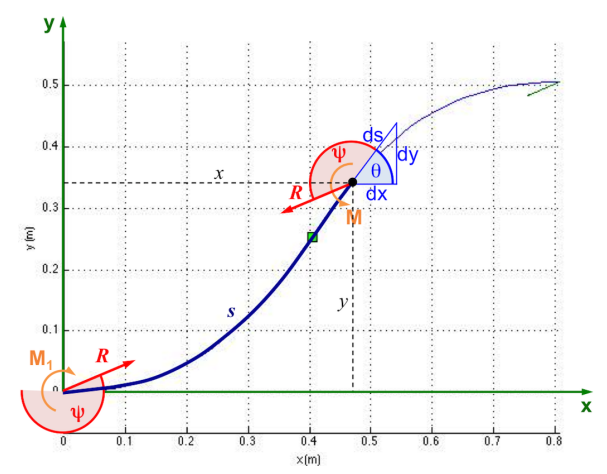

Fig. 1.1 A section of a planar bar at equilibrium

section in equilibrium (see Fig. 1.1): $R$ and $\psi$ as the force (modulus and orientation respectively) at the extremity, $M_{1}$ as the bending moment at a extreme, and $M$ as the bending moment at a cross-section. The static equilibrium of moments for that portion of bar can be expressed to get $M$ and substitute into Eq. 1.1 to obtain:

$$
\kappa=\frac{d \theta}{d s}=\frac{M}{E I}=\frac{M_{1}}{E I}+\frac{R}{E I} \cos \psi y-\frac{R}{E I} \sin \psi x
$$

Its derivative with respect to the arc length $s$ yields

$$
\frac{d \kappa}{d s}=\frac{d^{2} \theta}{d^{2} s}=\frac{R}{E I} \cos \psi \frac{d y}{d s}-\frac{R}{E I} \sin \psi \frac{d x}{d s}
$$

and in terms of $\theta$ it gives

$$
\frac{d \kappa}{d s}=\frac{d^{2} \theta}{d^{2} s}=\frac{R}{E I} \cos \psi \sin \theta-\frac{R}{E I} \sin \psi \cos \theta=\frac{R}{E I} \sin (\theta-\psi)
$$

A first integration in $\theta$ provides the expression 


$$
\frac{\kappa^{2}}{2}=-\frac{R}{E I} \cos (\theta-\psi)+C
$$

where $C$ is a constant of integration. As a result the following differential equation is obtained

$$
\kappa=\frac{d \theta}{d s}=\sqrt{2 C-\frac{2 R}{E I} \cos (\theta-\psi)}
$$

A second integration requires a more complex mathematical manipulation. Several approaches exist in the literature, here we will follow [5], where the first step is a sort of change of variables from $\theta, C$ to $\phi, k$ to give

$$
\begin{aligned}
C & =\frac{R\left(2 k^{2}-1\right)}{E I} \\
\cos \left(\frac{\psi-\theta}{2}\right) & =k \sin \phi
\end{aligned}
$$

Differentiation of Eq. (1.8) gives

$$
d \theta=\frac{2 k \cos \phi}{\sqrt{1-k^{2} \sin ^{2} \phi}} d \phi
$$

and simple trigonometric manipulation of Eq. (1.8) also provides

$$
\cos (\theta-\psi)=2 k^{2} \sin ^{2} \phi-1
$$

Upon substitution of Eqs. (1.7), (1.9) and (1.10) into Eq. (1.6), and further simplifications we get an integral from one extreme of the bar of length $L$ to the other:

$$
\sqrt{\frac{R L^{2}}{E I}}=\int_{\phi_{1}}^{\phi_{2}} \frac{1}{\sqrt{1-k^{2} \sin ^{2} \phi}} d \phi=F\left(k, \phi_{2}\right)-F\left(k, \phi_{1}\right)
$$

where $F(k, \phi)$ is the incomplete elliptic integral of the first kind.

For given boundary conditions on the slope of the bar at extremes, $\theta_{1}$ and $\theta_{2}$, and a given force at extremes $R$ and $\psi$, we can proceed as follows. Considering the case where $\theta_{1}$ and $\theta_{2}$ are null, it is obtained

$$
\begin{aligned}
\phi_{1}=\arcsin \left(\frac{1}{k} \cos \left(\frac{\psi}{2}\right)\right) & \phi_{2}=\phi_{1}+n \pi \\
\phi_{2} & =m \pi-\phi_{1}
\end{aligned}
$$

where $\phi_{1}=[-\pi / 2, \pi / 2], n=2,4, \ldots$ and $m=1,3, \ldots$ The angle $\phi$ will vary continuously from $\phi_{1}$ to $\phi_{2}$. Then, modulus $k=[-1,1]$ can be obtained iteratively on Eq. (1.11) for a certain value of $n$ or $m$. These later values define the buckling mode (see Fig. 1.2). Inflection points of the bar correspond to values $\phi=q \pi / 2$ with even values of $q$. 

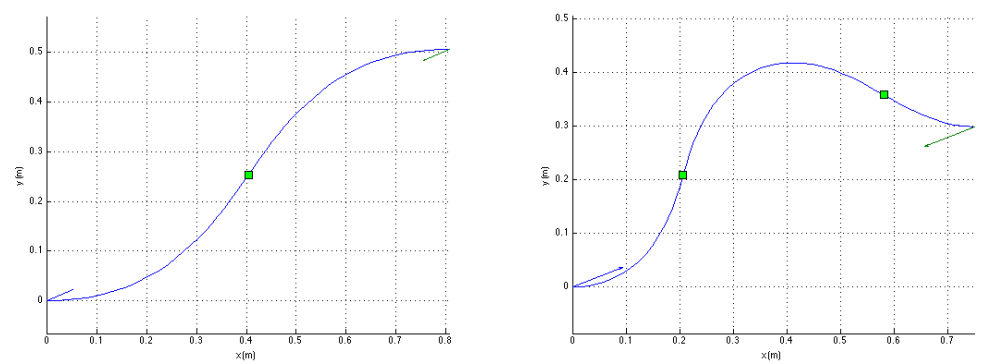

Fig. 1.2 Modes 1 and 2 of the buckling of a clamped-clamped bar

Upon substitution of Eqs. (1.7) and (1.8) into Eq. (1.6), the curvature at each point is given by

$$
\kappa=\frac{d \theta}{d s}=2 k \sqrt{\frac{R}{E I}} \cos \phi
$$

In order to get the $x$ coordinate of a point in the curve, Eq. (1.13) is expressed as

$$
\frac{d \theta}{d s} \frac{d x}{d x}=\cos \theta \frac{d \theta}{d x}=2 k \sqrt{\frac{R}{E I}} \cos \phi
$$

where we will substitute $d \theta$ by Eq. (1.9) and $\cos \theta$ as a function of $k$ and $\psi$ from Eq. (1.10). As a result we get the integral:

$$
\begin{aligned}
x= & -\sqrt{\frac{E I}{R}} \cos \psi\left[2 E\left(k, \phi_{i}\right)-2 E\left(k, \phi_{1}\right)-F\left(k, \phi_{i}\right)+F\left(k, \phi_{1}\right)\right]+ \\
& +\sqrt{\frac{E I}{R}} 2 k \sin \psi\left[\cos \phi_{i}-\cos \phi_{1}\right]
\end{aligned}
$$

where $E(k, \phi)$ is the incomplete elliptic integral of the second kind. In order to get the $y$ coordinate a similar procedure to the one followed for $x$ can be worked out.

For a given value of the coordinates of the extreme of the deflected bar, i.e. $a$ and $b$, and the boundary conditions on the slope of the bar at extremes $\theta_{1}, \theta_{2}$, we must iterate on $\psi$ and $k$ in Eq. (1.11), obtaining $x$ from Eq. (1.15) and and $y$ from an analogous equation, and verify the error with respect to $a$ and $b$ until it is below a given threshold.

From the above results we can note that solutions can be found between some limiting values for $k$ in an unknown range of $\psi$ for each mode separately. A minimum value for $k$, upon analysis of Eq. (1.12), corresponds to limiting values $\phi_{1}=-\pi / 2$ and $\phi_{1}=\pi / 2$. In the case of a clamped-clamped bar, modes 1 and 2 have a coincident $\phi_{2}=\pi-(-\pi / 2)=-\pi / 2+2 \pi$ and $\phi_{2}=\pi-(\pi / 2)=\pi / 2+2 \pi$. There is a common limit for both modes defined as the curve of end positions of the 
buckled bar for any $\psi$ and the limiting value of $k_{\min }=\left\|\cos \left(\frac{\psi}{2}\right)\right\|$, positive for the range $k=\left[k_{\min }, 1\right]$ and negative for the range $k=\left[-k_{\min },-1\right]$. If we limit the anal-
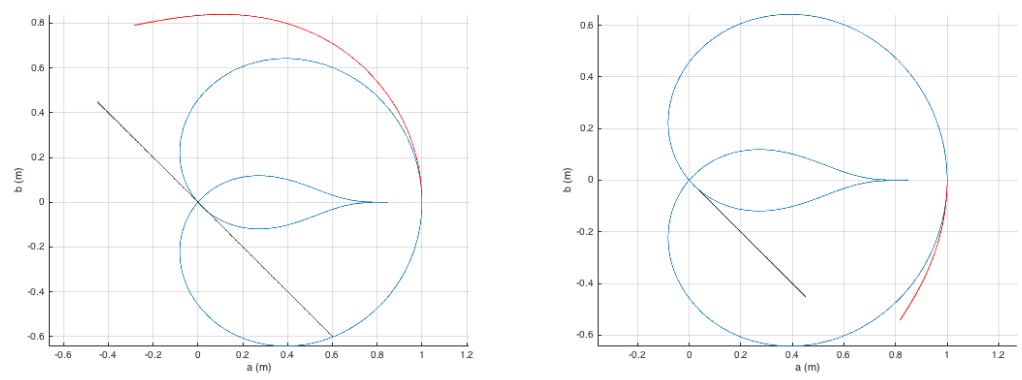

Fig. 1.3 Solutions for Modes 1(red curve) and 2 (black curve) for $\psi=135$ and $\psi=315$ from $k_{\text {min }}$ to $\mathrm{k}=1$

ysis to positive values of $k$, and plot the end positions of the bar for a given value of $\psi$ in the range from $k_{\min }$ to $k=1$ we get the plot in Fig. 1.3. As it can be seen, solutions for both modes start from the limiting curve of $k_{\min }$ and go up to a $k$ value of 1 .

\subsection{Kinematic Analysis of the 2 DoF Parallel Continuum Robot}

The case study is a planar parallel continuous robot with 2 degrees of freedom where two linear actuators introduce forces on two collinear sliders where bars have been clamped at 45 degrees, and are welded together at 90 degrees at the other extremity. From a home position where no deformation is forced, moving the sliders closer will make the end-effector reach upper positions, while getting away produces the contrary (see Fig. 1.4).

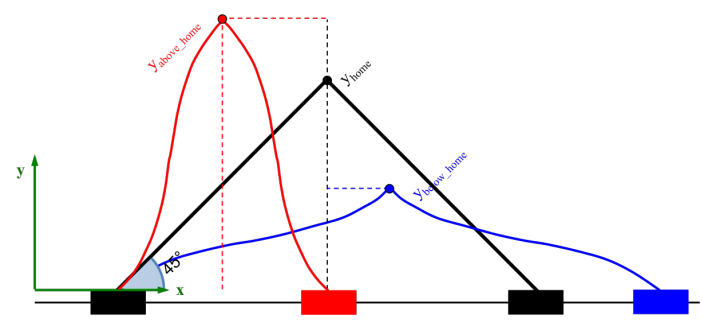

Fig. 1.4 Solutions of the Position Problem of a 2 DoF Parallel Continuum Robot 
The purpose of the kinematic analysis is to find the function between the value of such relative compression-extension of sliders and the $y$ coordinate reached by the end-effector point. In order to do that, the key point is the condition of force equilibrium to be accomplished. At any time the forces exerted by one bar onto the other at the end-effector connection must be in equilibrium. With no load applied it implies that a force $R$ is parallel to the sliding guide (see Fig. 1.5). Therefore, the angle $\psi$ of the force at the end of each of the clamped bars is known.

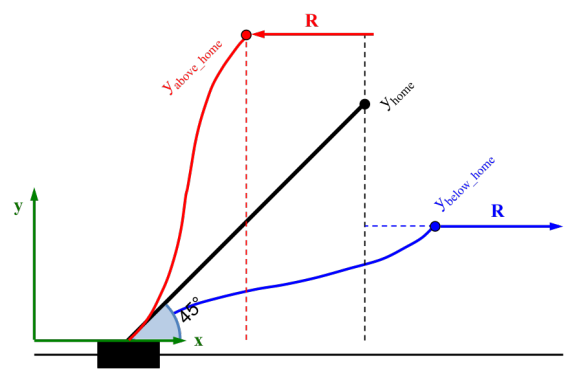

Fig. 1.5 Forces at equilibrium in such positions

If we rotate the problem in Fig. 1.545 degrees clockwise, we get the fundamental problem of a bar clamped at both extremes studied in the previous section. The solutions for the bar under compression will be on the curve of solutions for $\psi=135$ degrees, while for tension on the curve of $\psi=315$ degrees. Superimposing Fig. 1.5 on Fig. 1.3 we get Fig. 1.6. As mode 2 is out of the possible solutions, only curves corresponding to mode 1 are shown.

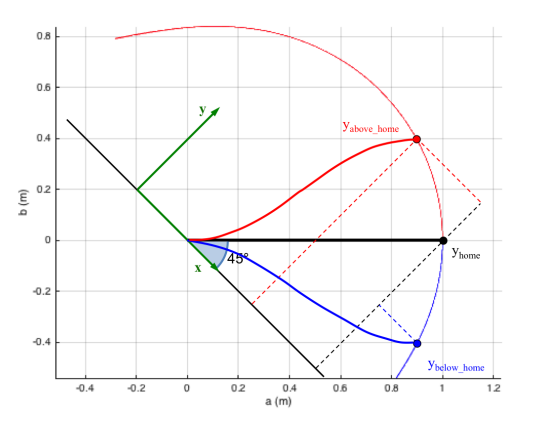

Fig. 1.6 Feasible solution Curve of the Position Problem

The computed plot is used to solve Inverse and Direct Position problems. In the Inverse Position Problem, for a given value of $y$ for the end-effector point, we draw 
a line parallel to the rotated $x$ axis at such a distance. At the intersection with the curve we get the feasible solution, either for compression or tension. In the Direct Position Problem, for a given value of the half-distance between sliders, we draw a line parallel to the rotated $y$ axis at such a distance from the first slider and at the intersection with the curve we get the solution.

\subsection{Numerical and Experimental Validation}

In order to validate the proposed analytical approach, a numerical analysis using FEA and an experimental analysis have been carried out. The FEM model consists of two beam elements clamped together at a right angle, with two specific boundary conditions. These conditions are two clamped ends at the non-common vertex of the beams. The material is PTFE with an elastic modulus of E=4 GPa. The model is analyzed using the non-linear method. Beam189-type elements have been used in the model by the software ANSYS. Starting from an initial configuration of the system where no deformation exists, forced displacements either in the input or the output are imposed. Solutions to both cases are identical. A simple prototype was assem-

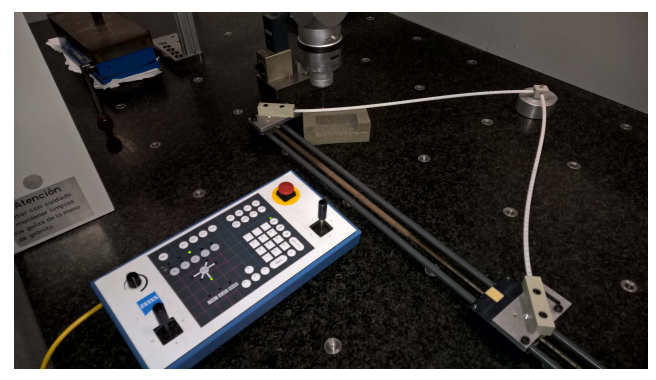

Fig. 1.7 The experimental set up with a prototype

bled and a series of experimental measurements were taken on an MC 850 ZEISS three-dimensional measuring machine (precision is $+-0.005 \mathrm{~mm}$ ), with experimental set up in Fig. 1.7. Results obtained using analytical method, FEM analysis, and experimental measurements are compared in the plots of Fig. 1.8. Validation of the analysis methods is accomplished.

\subsection{Conclusions and Future Work}

The use of flexible links to create parallel devices has a potential to produce new mechanisms for applications where human interaction is present. The analytical 


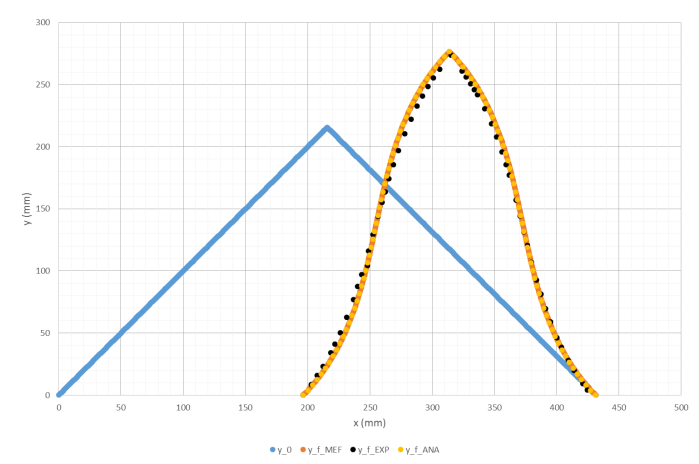

Fig. 1.8 Comparison of analytical, numerical and experimental solutions of the Position Problem

methods available in the literature can be adjusted to solve the position problems of such mechanisms under the introduction of additional conditions on the force equilibrium of the mechanism. A simple parallel continuum mechanism has been solved to prove the feasibility and implementation of the proposed approach for kinematics analysis and characterization of Continuum Parallel robots. Compared to tensegrity mechanisms, the number of elements in the system is minimized because of the capability of the rigid elements to work under compression mode.

Acknowledgements The authors wish to acknowledge the financial support received from the Spanish Government through the Ministerio de Economía y Competitividad (Project DPI201564450-R) and the Regional Government of the Basque Country through the Departamento de Educación, Universidades e Investigación (Project IT445-10) and UPV/EHU under program UFI $11 / 29$. Also, the support of ERASMUS program is gratefully acknowledged by the fourth author.

\section{References}

1. Bionic Tripod 3.0. Festo Innovation and Technology. http://www.festo.com

2. Bryson C. E. and Rucker D. C.: Toward Parallel Continuum Manipulators. IEEE International Conference on Robotics and Automation, 778-785, (2014).

3. Antman S.S.: Nonlinear Problems of Elasticity. Springer (2005) ISBN: 0-387-20880-1.

4. Howell, Larry L.: Compliant Mechanisms. Wiley (2001) ISBN: 978-0-471-38478-6.

5. Holst, G. L. et al.: Modeling and Experiments of Buckling Modes and Deflection of FixedGuided Beams in Compliant Mechanisms. Journal of Mechanical Design, 133, 051002-1-10 (2011)

6. Zhang, Aimei, and Guimin Chen: A comprehensive elliptic integral solution to the large deflection problems of thin beams in compliant mechanisms. Journal of Mechanisms and Robotics 5, 021006-1. (2013)

7. Tolley M. T. et al.: A resilient, Utethered Soft Robot. Soft Robotics, 778-223, (2014). 